\title{
Quantitative Characterization of Trabecular Bone Micro-architecture Using Tensor Scale and Multi-Detector CT Imaging
}

\author{
Yinxiao Liu, Punam K. Saha, and Ziyue Xu \\ Iowa Institute of Biomedical Imaging, Departments of ECE and Radiology \\ University of Iowa, Iowa City, IA, US 52242 \\ \{yinxiao-liu, punam-saha, ziyue-xu\}@uiowa.edu
}

\begin{abstract}
Osteoporosis, characterized by low bone mineral density (BMD) and micro-architectural deterioration of trabecular bone (TB), increases risk of fractures associated with substantial morbidity, mortality, and financial costs. A quantitative measure of TB micro-architecture with high reproducibility, large between-subjects variability and strong association with bone strength that may be computed via in vivo imaging would be an important indicator of bone quality for clinical trials evaluating fracture risks under different clinical conditions. Previously, the notion of tensor scale (t-scale) was introduced using an ellipsoidal model that yields a unified representation of structure size, orientation and anisotropy. Here, we develop a new 3-D t-scale algorithm for fuzzy objects and investigate its application to compute quantitative measures characterizing TB micro-architecture acquired by in vivo multi-row detector CT (MD-CT) imaging. Specifically, new measures characterizing individual trabeculae on the continuum of a perfect plate and a perfect rod and their orientation are directly computed in a volumetric BMD representation of a TB network. Reproducibility of these measures is evaluated using repeat MD-CT scans and also by comparing their correlation between MD-CT and $\mu$-CT imaging. Experimental results have demonstrated that the t-scale-based TB micro-architectural measures are highly reproducible with strong association of their values at MD-CT and $\mu$-CT resolutions. Results of an experimental mechanical study have proved these measures' ability to predict TB's bone strength.
\end{abstract}

Keywords: Trabecular bone, structural micro-architecture, quantitative geometry, tensor scale, skeletonization, CT imaging, biomechanics.

\section{$1 \quad$ Introducation}

Osteoporosis increases risk of fractures associated with substantial morbidity, mortality, and financial costs. Approximately, $30 \%$ of postmenopausal white women in the United States suffer from osteoporosis [1] and the prevalence in Europe and Asia is similar. Approximately one in two women and one in four men over age 50 will have an osteoporosis-related fracture in their remaining lifetime. Clinically, osteoporosis is defined by low bone mineral density (BMD). However, increasing evidence suggests that micro-architectural quality of trabecular bone (TB) is an important determinant of 
bone strength and fracture risk [2-5]. BMD only explains about $65 \%$ to $75 \%$ of the variance in bone strength [6,7], while the remaining variance is due to the cumulative and synergistic effect of various factors including bone macro- and microarchitecture, tissue composition, and micro-damage [8,9]. Therefore, a quantitative measure of TB micro-architecture with high reproducibility, large between-subjects variability and strong association with bone strength that may be computed via in vivo imaging would be an important indicator of bone quality for clinical trials evaluating fracture risks under different clinical conditions.

Saha et al. developed digital topological analysis (DTA) [10,11]which classifies surfaces (plates), curves (rods), junctions, and edges in a skeletal representation of a TB network using local topological parameters $[10,12,13]$. Although DTA is widely applied $[5,6,14,15]$, a major limitation of the method is that resulting classifications are inherently discrete failing to distinguish between narrow and wide plates. Later, Saha et al. developed volumetric topological analysis algorithm (VTA) [16] characterizing the topology of individual trabeculae on the continuum between a perfect plate and a perfect rod. Although VTA provides an effective measure of TB microarchitecture, its premise is built on digital topology and path propagation approaches and misses some important information related to structure orientation and anisotropy.

Here, a simultaneous solution to estimate TB plateness/rodness and orientation is presented using a geometric approach of representing local structures with tensor scale (t-scale) [17]; initial results of application of t-scale in TB micro-architectural analyses were reported in [18]. T-scale provides a parametric representation of local structures using an ellipsoidal model. A new 3-D t-scale algorithm is developed for fuzzy objects and its application is studied to characterize TB micro-architecture acquired by in vivo multi-row detector CT (MD-CT) imaging. Other applications of tscale may include assessment of vessel and airway wall geometry and detection and segmention of lung fissures in pulmonary CT imaging, and also, assessment of gyri and polyps geomtery in neuro-imaging and in virtyal colonoscopy, respectively. In the following section, we briefly present the algorithms for computation of t-scale and TB measures which will be followed by description of experimental plans and methods. Finally, we discuss the results and draw our conclusion.

\section{Methods and Algorithms}

\section{$2.1 \quad 3-D$ T-Scale Computation}

T-scale-based quantitative micro-architectural assessment algorithm may be applied on fuzzy representation of an object where the membership value at each image voxel is interpreted as local object content or density. Here, we intend to apply the algorithm on TB bone images where the value at each voxel $p$ represents the bone mineral content (BMC) at $p$ and is denoted by $B M C(p)$. In the rest of this section, fuzzy membership and BMC will be used synonymously. T-scale computation is performed by locally tracing an object along $m$ pairs of mutually opposite sample lines selected at an approximately uniform distribution over the entire 3-D angular space ensuring that the final t-scale is not skewed in any direction. Here, we have used $22.5^{\circ}$ of angular interval between every two neighboring sample lines. Interval length between two 
successive sample points on each sample line is chosen equal to image voxel resolution. The extent of a fuzzy object along an individual sample line is determined by tracing the first sample points where the interpolated membership value is zero and an edge point is located at that sample point location. These edge points are intended to roughly describe the boundary of the t-scale ellipse centered at the candidate voxel, denoted as $p$. Following the axial symmetry of an ellipse, for each pair of opposite sample lines, the edge points are repositioned so that each pair of two edge points on the opposite direction are equidistant from the center point $p$. Finally, an ellipsoid is fit to those repositioned edge points; in the rest of this section, "edge point" will refer to "repositioned edge point". The literature on ellipsoid fitting is quite mature where, essentially, an error between the observed data (here, the edge points) and the computed ellipsoid is minimized. Here, the geometric distance error is used and the algorithm is summarized as follows.

Step 1. Translate all edge points to move the candidate point $p$ at the origin.

Step 2. Apply principle component analysis (PCA) to edge points computing eigenvectors $\left(\mathbf{i}_{1}, \mathbf{i}_{2}, \mathbf{i}_{3}\right)$ and eigenvalues $\left(\lambda_{1}, \lambda_{2}, \lambda_{3}\right)$ of the point-distribution.

Step 3. Rotate the edge points to align $\mathbf{i}_{1}, \mathbf{i}_{2}$ and $\mathbf{i}_{3}$ with the three coordinate axes.

Step 4. Determine the smallest box enclosing all edge points and use it to determine the initial guess for the best-fit ellipsoid.

Step 5. Compute the final ellipsoid by applying iterative minimization of the sum of geometric distance errors using the Newton's optimization algorithm.

See Fig. 1 for illustration of steps in t-scale computation; here, 2-D illustrations are used to improve the quality of presentation.

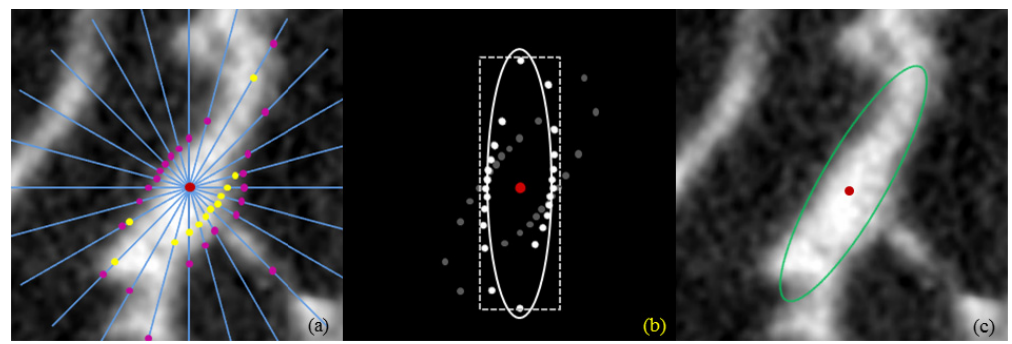

Fig. 1. Steps in t-scale computation. (a) Edge points (pink) on radial sample lines (blue) and repositioned edge points (yellow). (b) Bounding box (dotted) and the best-fit ellipse (grey) after rotating repositioned edge points (grey) using PCA. (c) Final t-scale ellipsoid (green).

\subsection{Trabecular Bone Quality Analysis}

As described in the previous section, t-scale produces three eigenvectors $\left(\mathbf{i}_{1}, \mathbf{i}_{2}, \mathbf{i}_{3}\right)$ and corresponding eigenvalues $\left(\lambda_{1}, \lambda_{2}, \lambda_{3}\right)$ parametrically representing local structures. The idea here is to use these parameters to characterize micro-architecture of individual trabeculae. Let $\mathbf{i}_{j}(p)$ and $\lambda_{j}(p)$, where $j=1,2,3$, denote the three eigenvectors and eigenvalues, respectively, at a given TB voxel $p$. The local structure width at $p$, denoted by $T S_{W}(p)$, may be defined in the millimeter unit using the length of the second largest 
eigenvalue $\lambda_{2}(p)$ as shown in Fig. 2b; local structure measure using the VTA algorithm is presented in in Fig. 2c which has been thorough evaluated in [16]. The agreement of the TB width measures using the two different approaches as shown in Fig. 2b,c is encouraging. The motivation of investigating the t-scale method is two-folded - (1) t-scale computes the local structure width measure using a geometric approach as compared to the VTA method based on digital topology and path propagation and (2) simultaneous measures of local structure orientation and thickness. Here, we study the orientation measure of TB. The orientation of the structure, denoted by $T S_{O}(p)$, is defined by the cosine of the angle between the eigenvector $\mathbf{i}_{1}(p)$ corresponding to the largest eigenvalue and the bone's longitudinal axis; color-coded illustration of the orientation measure is shown in Fig. 2e. As observed in the figure, the t-scale-based orientation measure successfully distinguishes between the longitudinal (green) and transverse (red) trabeculae. Also, the normalized plateness measure over the [0,1] interval, denoted by $T S_{P}(p)$, is classically defined using the anisotropy between the length of the second largest eigenvalue $\lambda_{2}(p)$ and the smallest eigenvalue $\lambda_{3}(p)$ as follows:

$$
T S_{P}(p)=\sqrt{1-\left(\lambda_{3}(p) / \lambda_{2}(p)\right)^{2}} .
$$

It may be noted that the above plateness measure does not require threshold values as needed in VTA [16]. The normalized rodness measure $T S_{R}(p)$ is defined as:

$$
T S_{R}(p)=1-T S_{P}(p)
$$

The above measures at an individual voxel location may be directly computed from its local t-scale. However, it can be shown that these measures suffer from edge artifacts when the target voxel is far from the skeleton due to the failure of covering the entire geometry of the local structure within its t-scale. Therefore, we define our algorithm as follows using an initialization and feature propagation approach.

1) Compute the surface skeleton $S$ for the TB structure $O$ where $O$ is set of all voxels with nonzero BMC value.

2) For each volxel $p \in S$, initiate the TB measures values: $T S_{W}(p), T S_{O}(p)$, $T S_{P}(p)$ and $T S_{R}(p)$ as defined above.

3) At each non-skeletal voxel $q \in O-S$, inherit the TB measures: $T S_{W}(q)$, $T S_{O}(q), T S_{P}(q)$ and $T S_{R}(q)$ from the nearest skeletal voxel $p$ using a feature propogation algorithm.

In the above steps, the surface skeletonization is computed using the algorithm by Saha et al. [19] and the noise removal procedure presented in [16]. The feauture propagation is accomplished using the classical algorithm introduced in [16]. Finally, the following TB measures are computed over a VOI $V$ as a parameter representing the micro-architectural properties of the TB over $V$ :

1. Bone mineral density: $B M D=\sum_{p \in V} B M C(p) /\|V\|$,

2. Surface width: $S W_{T S}=\sum_{p \in V} T S_{W}(p) B M C(p) / \sum_{p \in V} B M C(p)$,

3. Surface curve ratio: $S C R_{T S}=\sum_{p \in V} T S_{P}(p) B M C(p) / \sum_{p \in V} T S_{R}(p) B M C(p)$,

4. Longitude bone mineral density: $B M D_{\text {Long }}=\sum_{p \in V} T S_{O}(p) B M C(p) /\|V\|$,

5. Transverse bone mineral density: $B M D_{\operatorname{Tran}}=\frac{\sum p \in V\left(1-T S_{O}(p)\right) B M C(p)}{\|V\|}$. 


\section{Experimental Plans and Methods}

The experiment was performed on 15 fresh-frozen human cadaveric ankle specimens harvested from 11 body donors. Six steps were performed on each ankle specimen in the following order - (1) MD-CT imaging, (2) Soft-tissue removal and tibia dislocation, (3) $\mu$-CT imaging, (4) specimen preparation, (5) mechanical testing, and (6) image processing and computation of TB measurements. All ankle specimens were kept frozen until the performance of MD-CT imaging.

High resolution MD-CT scans of the distal tibia were acquired at the I-CLIC center, University of Iowa on a 128 slice SOMATOM Definition Flash scanner at $120 \mathrm{kV}, 200$ effective mAs, and reconstructed at $0.2 \mathrm{~mm}$ slice thickness using a special U70u kernel achieving high structural resolution. An INTable ${ }^{\mathrm{TM}}$ Calibration Phantom Couch Pad was scanned to calibrate CT Hounsfield units into BMC $\left(\mathrm{mg} / \mathrm{cm}^{3}\right)$. Three repeat MD-CT scans of each distal tibia specimen were acquired after repositioning the specimen on the CT table before each scan. Each specimen was also scanned on an Imtek Micro-cat II scanner at $28.8 \mu \mathrm{m}$ isotropic resolution after removing soft tissue and dislocating tibia from the ankle joint.

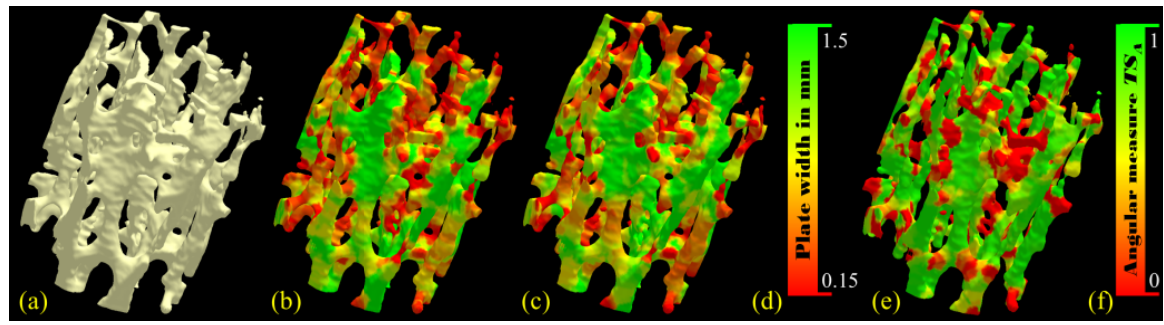

Fig. 2. Characterization of TB micro-architecture using the t-scale. (a) A TB region selected from $\mu$-CT image of a human ankle specimen. (b,c) TB characterization on the continuum between a perfect plate (green) and a perfect rod (red) using the t-scale- VTA-based algorithms. (d) Color-coding for (b,c). (e) TB orientation analysis using t-scale. (f) Color-coding for (e).

To determine actual TB strength, a cylindrical TB core of nominally $8 \mathrm{~mm}$ in diameter and $20.9 \pm 3.3 \mathrm{~mm}$ in length were cored from distal tibia in situ along the proximal-distal direction. The TB cores were mechanically tested in compression using an electromechanical materials testing machine. To minimize specimen end effects, strain was measured with a $6 \mathrm{~mm}$ gage length extensometer attached directly to the midsection of the bone. A compressive preload of $10 \mathrm{~N}$ was applied and strains then set to zero. At a strain rate of $0.005 \mathrm{sec}^{-1}$, each specimen was preconditioned to a low strain with at least ten cycles and then loaded to failure. Yield stress was determined as the intersection of the stress-strain curve and a $0.2 \%$ strain offset of the modulus.

Finally, each TB MD-CT data was processed through the following cascade of image processing steps - (1) computation of bone mineral content or BMC image using a stepup ramp function, (2) re-sampling of BMC images at $0.15 \mathrm{~mm}$ isotropic voxel using the linear interpolation method, (3) do surface skeletonization and (4) application of t-scale to each skeleton and propagate back to TB volume. MD-CT Hounsfield number at each voxel was converted to a BMC $(\mathrm{mg} / \mathrm{cc})$ value using average $\mathrm{MD}-\mathrm{CT}$ values in three calibration rods in the INTable ${ }^{\mathrm{TM}}$ Calibration Phantom at concentrations of 0,75 and 150 
$\mathrm{mg} / \mathrm{cc}$ of calcium hydroxyapatite homogeneously (CaHA) blended into the CTWater $^{\mathrm{TM}}$ compound. For a $\mu-\mathrm{CT}$ scan, bone volume fraction (BVF) image was used instead of BMC due to lack of a calibration phantom for $\mu-C T$. Therefore, BV/TV was computed over a VOI instead of BMD.

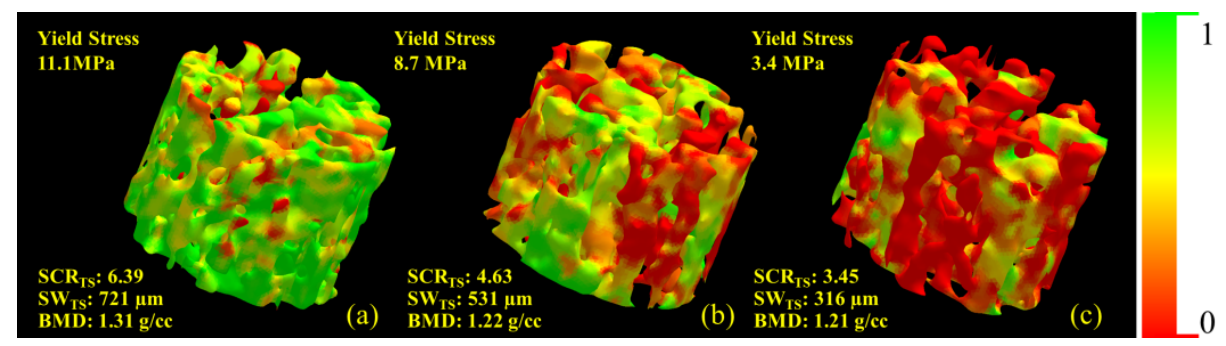

Fig. 3. Illustration of the t-scale plate measure for three different TB specimens - (a) strong (Yield stress: 11.1MPa), (b) moderate (7.1MPa) and (c) weak (3.4 MPa).

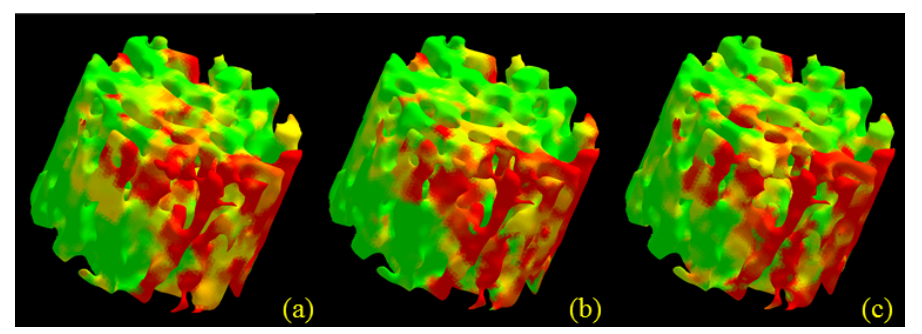

Fig. 4. Illustration of reproducibility for t-scale-based plateness classification of a TB specimen in three repeat MD-CT scans (a-c) shown using the same color coding scheme of Fig. 3.
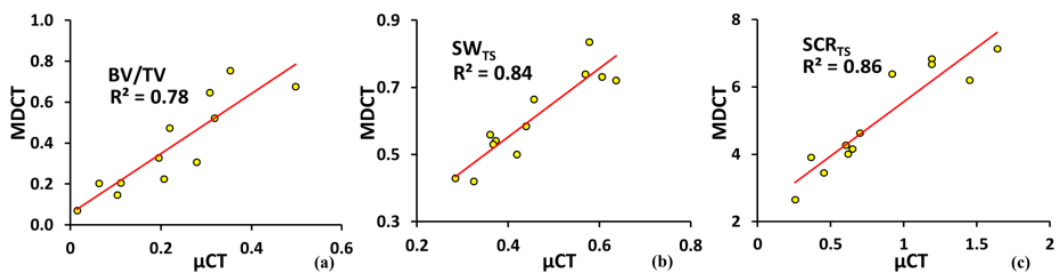

Fig. 5. Correlation of different TB measures, namely $\boldsymbol{B V} / \boldsymbol{T V}$ (a), $\boldsymbol{S} \boldsymbol{W}_{\boldsymbol{T} \boldsymbol{S}}$ (b) and $\boldsymbol{S C} \boldsymbol{R}_{\boldsymbol{T S}}$ (c), computed via MD-CT and $\mu-\mathrm{CT}$ imaging.
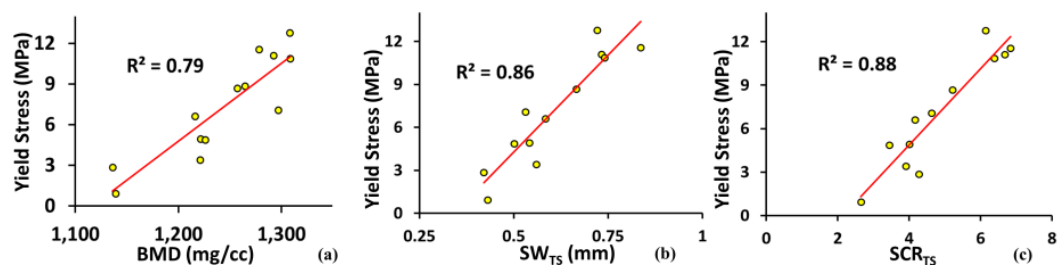

Fig. 6. Ability of different TB measures to predict bone strength shown in terms of $\mathrm{R}^{2}$ values of linear correlation between Yield stress and each of $\boldsymbol{B M D}$ (a), $\boldsymbol{S} \boldsymbol{W}_{\boldsymbol{T} S}$ (b) and $\boldsymbol{S C} \boldsymbol{R}_{\boldsymbol{T S}}$ (c). 


\section{$4 \quad$ Results and Conclusion}

Results of t-scale based classification of TB micro-architecture are illustrated in Fig. 2 and the plateness/rodness classification results are visually compared with VTA results. Results of t-scale-based plateness classification of TB network for three specimens with different experimental mechanical strengths are shown in Fig. 3. As shown in the figure, a $8 \%$ difference in BMD from a strong bone (a) to a weak bone (c) leads to $70 \%$ loss in bone strength and manifests into $60 \%$ difference in TB microarchitectural measure $S C R_{T S}$ establishing its high sensitivity in bone degeneration.

Repeat scan MD-CT reproducibility of the method under in vivo condition was determined for BMD and all five t-scale measures. Color-coded illustration of t-scale plateness measure over a matching region in three repeat scans MD-CT data is shown in Fig. 4. For this study, ten spherical VOIs each of $3.75 \mathrm{~mm}$ radius were randomly selected in the first MD-CT scan of each specimen above the position $8 \mathrm{~mm}$ proximal to the distal endplate leading to a total of 150 VOIs. Post-registration algorithm was used to locate the matching VOIs in the second and the third scans. Finally, the intraclass correlation (ICC) of three repeat scans was computed for each TB measure and the observed results are - (1) BMD: 0.997, (2) $\boldsymbol{B} \boldsymbol{M} \boldsymbol{D}_{\text {Long }}: 0.983$, (3) $\boldsymbol{B} \boldsymbol{M} \boldsymbol{D}_{\mathrm{Tran}}: 0.987$, (4) $S W_{V T A}: 0.966$ and (5) $S C R_{V T A}: 0.953$. Although the two t-scale-based measures $S W_{T S} S C R_{T S}$ have demonstrated slightly lower ICC values than the BMD measures, which are expected to be highly reproducible in $\mathrm{CT}$ imaging modality, the observed ICC values are satisfactory considering the fact that the measures were computed over small VOIs and less averaging of errors.

Linear correlations of three different TB measures derived from MD-CT and $\mu-C T$ imaging are graphically illustrated in Fig. 5. For these experiments, 15 VOIs were used from 15 specimens and same VOIs are used for correlation study with TB pressure experiments. The t-scale-based measure $S C R_{T S}$ shows higher linear correlation between in vivo (MD-CT) and ex vivo ( $\mu-\mathrm{CT})$ resolutions as compared to the BMD measure; note that BV/TV measure was used for $\mu-\mathrm{CT}$.

For correlation analysis with TB's experimental Yield stress, the image-based measures were computed over a cylindrical VOI with its axis aligned with that of distal tibia and its length and position were selected as per the data recorded during specimen preparation. The results of correlation analysis between Yield stress and different TB measures are shown in Fig. 6. Both t-scale measures have demonstrated better strength to predict TB's Yield stress as compared to BMD.

In this paper, we have developed a new 3-D t-scale algorithm for fuzzy object and have investigated its role in computing quantitative TB micro-architecture measures through MD-CT imaging under an in vivo condition. Results of an extensive study on fifteen cadaveric ankle specimens evaluating the new t-scale-based method are presented. Observed results have demonstration satisfactory repeat scan reproducibility of method. High correlation of t-scale measures derived via in vivo and ex vivo imaging modalities is observed. Also, t-scale-based TB micro-architectural measures have demonstrated higher ability to predict trabecular bone's experimental mechanical properties under an in vivo condition. 


\section{References}

[1] Melton, 3rd.: Epidemiology of spinal osteoporosis. Spine 22, 2S-11S (1997)

[2] Benito, et al.: Deterioration of trabecular architecture in hypogonadal men. J. Clin. Endocr. Metab. 88, 1497-1502 (2003)

[3] Chesnut, 3rd., et al.: Effects of salmon calcitonin on trabecular microarchitecture as determined by magnetic resonance imaging:results from the QUEST study. J. Bone Miner. Res. 20, 1548-1561 (2005)

[4] Kleerekoper, et al.: The role of three-dimensional trabecular microstructure in the pathogenesis of vertebral compression fractures. Cal. Tis. Int. 37, 594-597 (1985)

[5] Wehrli, et al.: In vivo magnetic resonance detects rapid remodeling changes in the topology of the trabecular bone network after menopause and the protective effect of estradiol. J. Bone Miner. Res. 23, 730-740 (2008)

[6] Wehrli, et al.: Role of magnetic resonance for assessing structure and function of trabecular bone. Topics in Mag. Res. Imag. 13, 335-356 (2002)

[7] Ammann, et al.: Bone strength and its determinants. Osteoporos Int. 14, S13-S18 (2003)

[8] Chapurlat, et al.: Bone microdamage: a clinical perspective. Ost. Int. 20, 1299-1308 (2009)

[9] Seeman, et al.: Bone quality-the material and structural basis of bone strength and fragility. N. Engl. J. Med. 354, 2250-2261 (2006)

[10] Saha, Chaudhuri: 3D digital topology under binary transformation with applications. Comp. Vis. Image Und. 63, 418-429 (1996)

[11] Saha, et al.: Three-dimensional digital topological characterization of cancellous bone architecture. Int. J. Imag. Sys. Tech. 11, 81-90 (2000)

[12] Saha, Chaudhuri: Detection of 3D simple points for topology preserving transformation with application to thinning. IEEE TPAMI 16, 1028-1032 (1994)

[13] Saha, et al.: Topology preservation in 3D digital space. Pat. Rec. 27, 295-300 (1994)

[14] Liu, et al.: Individual trabecula segmentation (ITS)-based morphological analysis of microscale images of human tibial trabecular bone at limited spatial resolution. J. Bone Miner Res. 26, 2184-2193 (2011)

[15] Chang, et al.: Adaptations in trabecular bone microarchitecture in Olympic athletes determined by 7T MRI. J. Mag. Res. Imag. 27, 1089-1095 (2008)

[16] Saha, et al.: Volumetric topological analysis: a novel approach for trabecular bone classification on the continuum between plates and rods. IEEE TMI 29, 1821-1838 (2010)

[17] Saha: Tensor scale: a local morphometric parameter with applications to computer vision and image processing. Com. Vis. Imag. Und. 99, 384-413 (2005)

[18] Saha, et al.: In vivo assessment of trabecular bone architecture via three-dimensional tensor scale. In: SPIE, pp. 750-760 (2004)

[19] Saha, et al.: A new shape preserving parallel thinning algorithm for 3D digital images. Pat. Rec. 30, 1939-1955 (1997) 